\title{
AN EXISTENCE THEOREM FOR NONLINEAR BOUNDARY VALUE PROBLEMS
}

\author{
KEITH SCHRADER ${ }^{1}$ AND PAUL WALTMAN ${ }^{2}$
}

This paper considers the two point boundary value problem

$$
\begin{aligned}
y^{\prime \prime}+f\left(t, y, y^{\prime}\right) & =0, \\
y(a) & =A, \\
y(b) & =B .
\end{aligned}
$$

Questions of the existence and uniqueness of solutions of (1) ,(2), (3) have a long mathematical history. It is often easy to prove that there exists at most one solution to this problem using differential inequalities or monotonicity considerations, but to prove that indeed a solution exists is often more difficult. (We note that "at most one solution" includes the case where "no solution" exists.) The theorem in this paper provides a set of circumstances where uniqueness-that is, the existence of at most one solution-implies existence of a solution.

The first results in this direction are those of Lasota and Opial [4] and Jackson [2] who, in addition to continuity of $f$ and the uniqueness and continuability of solutions of initial value problems, assumed that the boundary value problem

$$
y^{\prime \prime}+f\left(t, y, y^{\prime}\right)=0, \quad y\left(t_{1}\right)=x_{1}, \quad y\left(t_{2}\right)=x_{2}
$$

had at most one solution on $\left[t_{1}, t_{2}\right]$ for every $x_{1}, x_{2} \in R$ and all subintervals $\left[t_{1}, t_{2}\right]$ of $[a, b+\epsilon)$ (or of $\left.(a-\epsilon, b]\right)$. The requirement for uniqueness of solutions of initial value problems was removed independently by Schrader [5] and Waltman [9]. Schrader [5] also removed the condition for uniqueness of solutions on all subintervals but did require uniqueness for boundary value problems with endpoints in small intervals about $a$ and $b$. Shampine [7], by assuming something about the way uniqueness fails, removed the requirement for uniqueness in an interval about $a$. Bailey and Shampine [1] further improved this result, requiring at most one solution of (1), (2), $y\left(t_{2}\right)=x_{2}$, for $t_{2}$ in some small interval about $b$ and requiring existence and uniqueness of solutions of boundary value problems with both endpoints in this interval. The last two papers required uniqueness of solutions of the initial value problem and [1] in particular made strong use of continuity with respect to initial conditions.

Received by the editors June $10,1968$.

1 Supported by NSF grant GP 9015.

2 Supported by NSF grant GP 8173. 
We improve on these results by requiring, in addition to the continuity of $f$ and the continuability of solutions, only that there is at most one solution of (1), (2) and $y\left(t_{2}\right)=x_{2}$ for all $x_{2} \in R$ and $t_{2}$ $\epsilon(b-\epsilon, b+\epsilon)$ for some $\epsilon>0$. No further existence or uniqueness is required for boundary value problems; no uniqueness of solutions or continuous dependence on initial conditions for initial value problems is used in the proof.

THEOREM. Let $f\left(t, y, y^{\prime}\right)$ be continuous on $[a, b+\epsilon) \times R \times R$, where $\epsilon>0$, and suppose that all solutions of initial value problems for equation (1) exist on $[a, b+\epsilon)$. Assume that for a fixed $A$, there do not exist two solutions on $\left[a, t_{2}\right]$ to any of the problems (1), (2), $y\left(t_{2}\right)=x_{2}, x_{2} \in R$, $t_{2} \in(b-\epsilon, b+\epsilon)$. Then there exists exactly one solution of (1), (2), (3) for every $B$.

Proof. Denote by $y(t, m)$ any solution of (1), (2) and $y^{\prime}(a)=m$, and by $\{y(t, m)\}$ the set of points "reached" at $t$ by all solutions of (1), (2) and $y^{\prime}(a)=m$.

For $m \in R$ define $\Gamma(m)=\{y(b, m)\}$; since we are not assuming uniqueness of solutions of the initial value problem this is a point to set mapping. To determine properties of this mapping it is convenient to think of it as a composite $P F(m)$, where $F(m)$ is the funnel section at $t=b$ of the system corresponding to (1) and $P$ is the projection onto the $y$-axis. The funnel section of a compact connected set is compact and connected under our hypothesis that all solutions extend to $[a, b+\epsilon)$. This can be shown using the form of Kneser's Theorem given in Kamke [3] and is stated for autonomous systems in Sell [6, Theorem 5, p. 386]. Since $P$ is continuous it follows that $\Gamma$ takes compact connected subsets of the real line into compact connected subsets and thus $\Gamma(R)$ is connected. If $m_{1} \neq m_{2}$ then $\Gamma\left(m_{1}\right) \cap \Gamma\left(m_{2}\right)=\varnothing$ under the hypothesis of uniqueness of solutions to the boundary value problem. In this case, since $\Gamma(m)$ is connected, we order the subsets, $\Gamma(m)$, of the real line by $\Gamma\left(m_{1}\right) \leqq \Gamma\left(m_{2}\right)$ if and only if $\max \Gamma\left(m_{1}\right) \leqq \max \Gamma\left(m_{2}\right)$. Thus we can speak of $\Gamma$ being monotone.

We use the following lemma.

LEMma. Under the hypothesis of the theorem $\Gamma(m)$ is a monotone function of $m$.

Proof. We give a proof by contradiction. Let $m_{1}<m_{2}<m_{3}$ and $\Gamma\left(m_{1}\right)<\Gamma\left(m_{3}\right)<\Gamma\left(m_{2}\right)$ (as observed above $\Gamma\left(m_{i}\right)$ for $i=1,2,3$ are pairwise disjoint). $\Gamma[\alpha, \beta]$ is a connected set for every interval $[\alpha, \beta]$. Let $x_{i} \in \Gamma\left(m_{i}\right)$ and $x_{i}\left(t, m_{i}\right)$ the corresponding solution of (1), (2) and 
$x\left(b, m_{i}\right)=x_{i} . \Gamma\left[m_{1}, m_{2}\right]$ is connected and since $x_{1}<x_{3}<x_{2}$, there exists an $m^{*} \in\left[m_{1}, m_{2}\right]$ such that $x\left(b, m^{*}\right)=x_{3}$. Since $x\left(b, m_{3}\right)=x_{3}$ and $m^{*} \leqq m_{2}<m_{3}$, this contradicts uniqueness. The other case follows similarly. (Of course, if $\Gamma(m)$ is monotone and point valued, then solutions are unique. For an investigation of uniqueness and monotonicity see Sherman [8].)

If $\Gamma(R)=R$ then (1), (2), (3) has a solution for every choice of $B$. First of all $\Gamma(R)$ is open, for if there existed a point $\eta \in \Gamma(R)$ which was not interior it must be an extreme point and the choice of a slope at $a$ either above or below (depending on the monotonicity) the slope of the solution of (1), (2), $y(b, m)=\eta$ produces a contradiction.

We will now show that $\Gamma(R)=R$. Assume that $\Gamma(R)$ is bounded above since the argument for $\Gamma(R)$ bounded below is similar. Let $\eta=\sup \Gamma(R)$. We use an argument of Lasota and Opial [4] to choose a sequence of initial conditions.

Let $x_{n}\left(t, m_{n}\right)$ denote a sequence of solutions of (1), (2) such that $x_{n}\left(b, m_{n}\right) \uparrow \eta$ and let $c \in(b, b+\epsilon)$. Then

$$
\frac{x_{n}\left(c, m_{n}\right)-x_{n}\left(b, m_{n}\right)}{c-b} \geqq \frac{x_{1}\left(c, m_{1}\right)-x_{n}\left(b, m_{n}\right)}{c-b} \geqq K
$$

where

$$
K=\min \left\{0, \frac{x_{1}\left(c, m_{1}\right)-\eta}{c-b}\right\}
$$

We can assume that for infinitely many $n, x_{n}^{\prime}\left(b, m_{n}\right) \leqq 0$ for a similar argument can be made for $c \in(b-\epsilon, b)$ in the opposite case.

Let

$$
S_{n}=\left\{t: 0 \geqq x_{n}^{\prime}\left(t, m_{n}\right) \geqq K, t \in[b, c]\right\} .
$$

This set is not empty by the mean value theorem. Let $s_{n}=\min S_{n}$ ( $S_{n}$ is closed). Now $s_{n}$ lies in a bounded set so we can choose a convergent subsequence $s_{n} \rightarrow s_{0}$ and a subsequence of that so $x_{n}^{\prime}\left(s_{n}, m_{n}\right) \rightarrow x_{0}^{\prime}$. By the definition of $s_{n}, x^{\prime}\left(t, m_{n}\right) \leqq 0$ in $\left[b, s_{n}\right]$ so that $x_{n}\left(s_{n}, m_{n}\right) \leqq \eta$ and since $x_{n}\left(s_{n}, m_{n}\right) \geqq \min \left\{x_{1}\left(t, m_{n}\right): t \in[b, c]\right\}$, we can also choose a subsequence of the above subsequence of $s_{n}$ such that $x_{n}\left(s_{n}, m_{n}\right) \rightarrow x_{0}$.

Let $S(\delta)$ be a $\delta$-ball in $E^{3}$ about $\left(s_{0}, x_{0}, x_{0}^{\prime}\right), \delta<b+\epsilon-s_{0}$. For large $n,\left(s_{n}, x_{n}\left(s_{n}, m_{n}\right), x_{n}^{\prime}\left(s_{n}, m_{n}\right)\right) \in S(\delta)$. Since all solutions can be continued over $[a, b+\epsilon)$, there exists $H$ such that $\left|x_{n}^{\prime}\left(t, m_{n}\right)\right|<H$, $t \in\left[a, s_{0}+\delta\right][10$, Theorem 3.7, p. 16]. Let $y(t, H)$ and $y(t,-H)$ be solutions of (1), (2). By the monotonicity of $\Gamma(m)$, either $y(b,-H)$ $>x_{n}\left(b, m_{n}\right) \rightarrow \eta$ so $y(b,-H) \geqq \eta$ or else $y(b, H)>x_{n}\left(b, m_{n}\right) \rightarrow \eta$ so $y(b, H)$ $\geqq \eta$. In either case we have a contradiction. 
A similar theorem can be proved using $(a-\epsilon, b]$ instead of $[a, b+\epsilon)$ and using $a_{0} y(a)+a_{1} y^{\prime}(a)=A$ instead of (2). These involve no new ideas.

\section{REFERENCES}

1. P. B. Bailey and L. F. Shampine, Existence from uniqueness for two point boundary value problems, J. Math. Anal. Appl. (to appear).

2. L. Jackson, "Subfunctions and second order differential inequalities" in Advances in mathematics, Academic Press, New York, 1968, pp. 307-363.

3. E. Kamke, Zur Theorie der Systeme gewöhnlicher Differentialgleichungen. II, Acta. Math. 58 (1932), 57-85.

4. A. Lasota and Z. Opial, On the existence and uniqueness of solutions of a boundary value problem for an ordinary differential equation, Colloq. Math. 18 (1967), 1-5.

5. K. Schrader, Existence theorems for second order boundary value problems, J. Differential Equations (to appear).

6. G. R. Sell, On the fundamental theory of ordinary differential equations, J. Differential Equations 1 (1965), 370-392.

7. L. Shampine, Existence and uniqueness for nonlinear boundary value problems, J. Differential Equations (to appear).

8. T. L. Sherman, Uniqueness for second order boundary value problems, J. Differential Equations (to appear).

9. P. Waltman, Existence and uniqueness of solutions to a nonlinear boundary value problem, J. Math. Mech. 18 (1968), 585-586.

10. T. Yoshizawa, Stability theory by Liapunov's second method, Publ. Math. Soc. Japan, No. 9, Tokyo, 1966.

UNIVERSITY OF MISSOURI AND UNIVERSITY OF IOWA 